\title{
Penser les paysages avec les trois synthèses du temps de Deleuze
}

James Kelly

Journal of French and Francophone Philosophy - Revue de la philosophie française et de langue française, Vol XXIX, No 1-2 (2021) pp 130-147

\author{
Vol XXIX, No 1-2 (2021) \\ ISSN 1936-6280 (print) \\ ISSN 2155-1162 (online) \\ DOI $10.5195 /$ jffp. 2021.965 \\ www.jffp.org
}

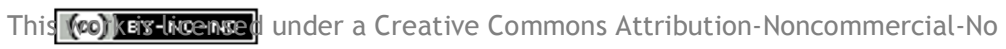
Derivative Works 3.0 United States License.

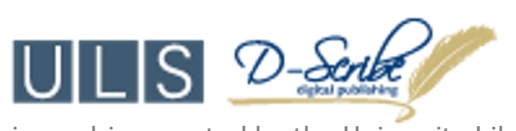

This journal is operated by the University Library System of the University of Pittsburgh as part of its D-Scribe Digital Publishing Program, and is co-sponsored by the University of Pittsburgh Press 


\title{
Penser les paysages avec les trois synthèses du temps de Deleuze
}

\author{
James Kelly \\ Independent Scholar
}

Ces dernières décennies, le rôle des êtres humains dans le monde naturel est devenu un thème fondamental qui touche tous les domaines de la connaissance. Nous sommes de plus en plus conscients des conséquences de notre mode de vie sur la Terre et ses écosystèmes ainsi que des dangers que cette situation représente pour la survie de notre espèce ${ }^{1}$. Sur le plan philosophique, l'œuvre de Pierre Montebello approfondit cette problématique, surtout dans les deux livres L'autre métaphysique et Métaphysiques cosmomorphes. Le premier de ces livres nous situe dans le contexte de la faille ouverte entre l'être humain et le monde naturel au cours du XVIII siècle ${ }^{2}$, qui a mené à la mécanisation de la nature et à sa domination par les humains. L'analyse de Montebello cherche à démontrer comment l'univocité au cœur des philosophies d'Henri Bergson et de Friedrich Nietzsche ouvre la voie à une métaphysique capable de surmonter ce schisme et de «refonder l'expérience [...] de l'unité originaire de l'homme et du $\operatorname{cosmos}^{3}$ ». Le second livre mentionné ci-dessus prolonge cet effort, analysant l'œuvre d'anthropologues contemporains tels que Philippe Descola, Eduardo Viveiros de Castro et Déborah Danowski, révélant ainsi une façon de penser qui va au-delà des philosophies anthropocentriques pour " recomposer un monde sans exclusive humaine et sans exclusion de l'homme ${ }^{4}$ ».

Nous examinons ici cette problématique en profondeur à partir de la perspective de la temporalité, en cherchant à montrer comment la philosophie du temps exposée par Gilles Deleuze dans Différence et répétition ${ }^{5}$ peut contribuer à recomposer un monde où l'être humain fait partie intégrante de la nature et à refonder ainsi notre expérience du cosmos. Grâce à la forte influence de Bergson et de Nietzsche sur sa philosophie du temps, nous verrons comment la métaphysique de Deleuze continue la lignée d'une " autre métaphysique » qui cherche à "retrouver le flux créateur des forces qui parcourt les choses et pouvoir mettre l'homme à l'unisson d'une 
puissance qui fulgure dans le monde $^{6} »$. Nous utiliserons le concept de «paysage » pour voir comment le temps deleuzien nous sert à penser les paysages - y compris ses habitants humains - comme élément intégral, non pas en termes d'espace ou d'étendue mais de perspective temporelle : il n'est pas question de chercher des frontières ni de délimiter ou de décrire une région de l'espace, il s'agit plutôt de révéler son être temporel en identifiant un ensemble de processus qui peuvent refléter certains de ses caractéristiques ; c'est-à-dire, de construire et de lire une " chronopédie $^{7}$ ». Cette lecture «chronopédique » nous révélera chaque paysage comme une composition de rythmes à différentes échelles : des processus géologiques qui durent des millions d'années jusqu'à d'autres, beaucoup plus courts, comme celui de l'habitation humaine. Cette lecture nous offrira aussi une nouvelle perspective du temps deleuzien. En la libérant des termes humains auxquels elle est exposée, elle révèle son potentiel pour résoudre l'écart entre le monde humain et la nature, et pour penser le monde au-delà de la perspective anthropocentrique qui a marqué plusieurs siècles de notre pensée.

\section{Le temps selon Différence et répétition}

Cet article procédera au moyen d'une lecture détaillée des trois synthèses décrites dans le chapitre «La répétition pour elle-même » dans Différence et répétition ${ }^{8}$, sur la base d'exemples empruntés à divers paysages du Chili pour démontrer l'application de chacune d'entre elles ${ }^{9}$. Cependant, avant de commencer, il nous faut faire quelques remarques sur le temps deleuzien. Notre commentaire sera soutenu par des contributions d'autres spécialistes, notamment la lecture minutieuse de James Williams, qui, comme d'autres auteurs, souligne l'importance et l'originalité de la pensée de Deleuze relative au temps ${ }^{10}$. C'est une théorie qui puise dans les écrits de nombreux philosophes, dont les idées de Plotin et de Hume relatives à la contemplation et à l'habitude, la pensée de Bergson relative à la durée et à la mémoire, l'éternel retour de Nietzsche, et la fracture kantienne du soi qui révèle la forme «pure » du temps. Comme l'a souligné Williams, la plus grande partie de l'originalité de la théorie de Deleuze réside dans sa capacité à recombiner ces éléments dans un cadre puissant et pluriel pour penser le temps.

Concrètement, la théorie du temps présentée dans Différence et répétition comprend trois synthèses qui abordent chacune un aspect différent de la temporalité. La première, le «présent vivant », est une synthèse passive qui contracte des instants répétés pour donner des présents finis ou intratemporels. Ce sont les habitudes de cette contraction qui constituent la fondation du temps et l'essence de l'être deleuzien. Ici, il faut remarquer que l'accent mis sur la répétition dans cette première synthèse - et d'une manière plus générale dans toute la métaphysique de Deleuze - est bien adapté à la description des paysages, émaillés partout de cycles et de rythmes. Cette première synthèse est complétée par une deuxième, le « passé pur », elle aussi passive mais qui constitue le fondement $\mathrm{du}$ temps en garantissant sa 
continuité, et par une troisième et dernière synthèse, cette fois active, qui explique le changement et la création du futur.

Examiner ces trois synthèses et réfléchir aux relations qu'elles entretiennent entre elles nous permet de comprendre la complexité et l'intangibilité du temps chez Deleuze. Les trois types de répétition, chacun avec ses dimensions correspondantes (le présent, à titre d'exemple, contient comme dimensions le passé et le futur), constituent un système où il n'existe pas une seule perspective unifiée du temps, ce qui amène Williams à parler d'une théorie fondée sur des temps «fracturés et disloqués » qui produisent un effet comme dans "une salle des miroirs brisés ${ }^{11}$ ». C'est cette nature «kaléidoscopique» de la théorie de Deleuze qui lui fournit sa grande puissance, particulièrement en ce qui concerne les paysages, avec sa myriade d'éléments, de synthèses, de rythmes et d'agents. Cette théorie de Deleuze nous donne un fondement métaphysique pour penser les phénomènes divers qui affectent et produisent les paysages, depuis leur géologie jusqu'à nous, les êtres humains qui les habitons.

\section{La fondation : le présent vivant}

Deleuze caractérise la première synthèse - le «présent vivant »-comme la fondation du temps. Cette synthèse passive, qui puise dans les analyses de Hume et de Bergson relatives à la répétition, fonctionne par la contraction d'une série d'instants répétés qui sont contemplés par un esprit. Cette contraction constitue un présent vivant qui comprend comme dimensions le passé ainsi que le futur. Il est important de souligner que ce n'est pas la série d'instants elle-même qui fonde le temps mais sa synthèse ou contraction, opération qui a lieu dans l'esprit et n'est pas faite par lui. Il est également important de bien comprendre ce que veut dire le terme «contracter » dans ce contexte. Dans sa définition de la première synthèse, Deleuze emploie le mot dans deux sens : le premier provient de Hume et décrit la formation des habitudes ; le deuxième est pris dans le sens bergsonien du mot, selon lequel la synthèse unit des instants et, par ce moyen, produit entre eux la durée. De la même façon, le mot «habitude » est employé par Deleuze dans un sens particulier: il ne s'agit pas seulement des «habitudes sensori-motrices que nous avons », mais il comprend « les habitudes primaires que nous sommes, les milliers de synthèses passives qui nous composent organiquement ${ }^{12}$ ».

Il faut souligner aussi que les contractions et les habitudes de cette composition de synthèses passives ne sont pas seulement confinées aux synthèses perceptuelles de notre esprit ou de notre perception psychologique du temps. Même si les synthèses qui constituent la fondation du temps dans notre conscience sont perceptuelles, Deleuze soutient qu'elles dépendent des innombrables synthèses organiques du corps humain qui constituent «la sensibilité du sens » : " il faut attribuer une âme au cœur, aux muscles, aux nerfs, aux cellules, mais une âme contemplative dont tout le rôle est de 
contracter $l^{\prime}$ habitude ${ }^{13} »$. En outre, le fonctionnement de cette synthèse n'est pas exclusif du corps humain : on le retrouve aussi, par exemple, dans le cas des plantes, puisqu' « il y a une contraction de la terre et de l'humidité qu'on appelle froment ${ }^{14}$ » et dans le cas général des organismes, Deleuze posant la question de savoir «quel organisme n'est pas fait d'éléments et de cas de répétition, d'eau, d'azote, de carbone, de chlorures, de sulfates contemplés et contractés, entrelaçant ainsi toutes les habitudes par lesquelles il se compose ${ }^{15}$ ». Dans sa présentation, l'explication de ces synthèses organiques est simplifiée, décrite, comme les exemples précédents, soit en termes d'éléments primitifs, comme l'eau, la terre, la lumière ou l'air, soit en termes d'éléments chimiques et composés, comme l'azote, le carbone, les chlorures et les sulfates. Néanmoins, cela nous révèle que cette première synthèse n'est pas seulement applicable à l'être humain dans ses strates psychiques et physiologiques mais aussi à l'être en général dans ses trois strates: physique, physiologique et psychique $^{16}$. Tout, soutient Deleuze, est contemplation, « même les rochers et les bois, les animaux et les hommes ${ }^{17}$ ».

Prenons un exemple de chacune de ces strates pour mieux comprendre le rapport entre cette première synthèse et les paysages. En premier lieu, au niveau physique - c'est-à-dire au niveau de la géosphère - prenons l'exemple des montagnes, spécifiquement les Andes du Chili. Cette orogénie s'est produite dans le contexte d'un processus de subduction dans lequel la plaque tectonique de Nazca rencontre la plaque sud-américaine, donnant lieu à une forte activité sismique qui a perduré pendant des dizaines de millions d'années. Ici, nous pouvons parler de la répétition des tremblements de terre provoqués par cette subduction et par les changements de forme et de configuration de la croûte. Dans le temps deleuzien, cette série de répétitions est contractée dans la première synthèse pour constituer un présent dans lequel les montagnes sont des exemples des habitudes de l'être du paysage. Nous pouvons également constater que dans ce présent, le passé est retenu dans la configuration de la Terre et qu'il y a aussi un futur dans la mesure où sa configuration vient à « anticiper » les tremblements et les mouvements des plaques dans une certaine direction.

En deuxième lieu, au niveau physiologique de la biosphère, on peut prendre l'exemple d'une forêt comme celles qui font partie des paysages du sud du Chili. Parmi les répétitions impliquées dans ce cas, il y a la répétition annuelle de la série des saisons, qui détermine la croissance des forêts. En contemplant cette répétition des éléments - les précipitations qui fournissent l'eau et le soleil qui font mûrir les fruits, permettant ainsi la reproduction des arbres - le paysage contracte cette série dans une synthèse pour constituer le présent de la forêt. Cette contraction définit aussi une habitude du paysage la forêt elle-même - qui s'ajoute à l'habitude sous-jacente des montagnes définies par le processus de subduction. En outre, dans ce présent, il y a aussi un passé retenu dans l'espèce des arbres ainsi qu'un futur dans la mesure où, 
dans cette habitude, le paysage attend ou anticipe les cycles saisonniers qui soutiennent la croissance continue de la forêt.

En troisième et dernier lieu, en ce qui concerne l'élément psychique $q^{\prime} u^{\prime}$ apportent les êtres humains faisant partie des paysages, prenons à titre d'exemple le réseau des sentiers préhispaniques qui traversent le désert au nord du Chili. Cette région est une des zones les plus sèches du monde, la surface de la Terre peut y rester quasiment inchangée pendant très longtemps. $C^{\prime}$ est un fait qui est mis en évidence par la présence des géoglyphes créés par les peuples préhispaniques à partir de différentes couleurs de pierres ${ }^{18}$. Ces formes curieuses se trouvent dans les pentes des collines sur les routes traversées par les caravanes de lamas utilisés par les peuples autochtones pour le transport de biens entre les oasis du désert et d'autres lieux d'importance pour l'habitation humaine. On peut parler ici d'un présent constitué par la contemplation et la contraction par le paysage des passages répétés des bêtes de somme; les sentiers tracés par leurs pas forment une habitude qui de nouveau fait partie de l'être du paysage. Mais il est également possible d'identifier un autre présent dans l'histoire de ces chemins : des années plus tard, vers la fin du XIX ${ }^{\mathrm{e}}$ siècle et au début du suivant, ces sentiers ont été repris pour le transport du salpêtre à l'époque où la consommation était en plein essor, cette fois à cheval. Encore une fois, on peut voir comment ce paysage a contemplé les traces laissées par l'activité humaine, comme il le fait à nouveau, dans un troisième présent où les archéologues et les visiteurs suivent les voyages des peuples préhispaniques, attirés par les mystères du désert. Cette succession de présents nous fournit un exemple pertinent de la façon dont « deux présents successifs peuvent être contemporains d'un même troisième, plus étendu par le nombre d'instants qu'il contracte ${ }^{19}{ }^{\prime}$.

Il y a deux remarques supplémentaires à propos de la première synthèse. La première nous renvoie à l'idée de composition, ou de chronopédie, que nous avons vue dans l'introduction de cet article. Dans son présentation, Deleuze parle de «nos mille habitudes composantes » et des « mille témoins qui contemplent en nous" et il remarque que "même dans le rat du labyrinthe, et dans chaque muscle du rat, il faut mettre de ces âmes contemplatives $^{20}$ ». De la même façon, dans les exemples ci-dessus, nous pouvons déjà voir comment, bien que d'une façon abstraite et schématique, la composition des diverses habitudes (la cordillère, la forêt, les sentiers créés par l'habitation humaine) définit un paysage par les présents qui le composent. La deuxième remarque concerne les rythmes. Cette première synthèse est par définition « intratemporelle » : elle constitue le présent « dans le temps », c'est-à-dire que c'est un présent qui possède « une certaine durée [...] variable suivant les espèces, les individus, les organismes et les parties d'organisme considérées » et qui passe ${ }^{21}$. Dans les exemples, nous avons vu des présents faits de différentes durées superposées : la durée relativement courte des périodes d'habitation humaine du désert qui n'ont perduré que 
quelques siècles ou moins et la durée des forêts à l'échelle de milliers d'années, jusqu'à la durée des montagnes à l'échelle de millions d'années. Cette temporalité est révélée à partir d'une lecture chronopédique, aidée par diverses disciplines scientifiques (la géologie, l'écologie, l'archéologie) qui nous permettent de déchiffrer l'histoire de cette myriade de présents ainsi que les habitudes correspondantes.

\section{Le fondement : le passé pur}

La nature finie ou intratemporelle du présent constitué par la première synthèse implique que ce présent doit se terminer. Par conséquent, déduit Deleuze, il doit exister une deuxième synthèse - le fondement de cet acte de fondation - qui constitue « un autre temps dans lequel s'opère la première ${ }^{22}$ ». Son exposition de cette deuxième synthèse puise beaucoup dans ses études sur la philosophie de Bergson, où il a décrit un « passé en général qui n'est pas le passé particulier de tel ou tel présent, mais qui est comme un élément ontologique, un passé éternel et de tout temps, condition pour le "passage" de tout présent particulier ${ }^{23}$ ». Dans ce « passé en général », la temporalité du présent et du passé est renversée : «à la limite, les déterminations ordinaires s'échangent : c'est du présent qu'il faut dire à chaque instant déjà qu'il "était", et du passé qu'il "est" 24 ». L'évocation de la limite ici - un concept qui fait allusion à l'importance du calcul infinitésimal dans la pensée de Deleuze relative à la différence ${ }^{25}$ - est capitale et nous fournit une façon d'appréhender le fonctionnement de la deuxième synthèse. Au fond, il s'agit du «mouvement » du temps, permettant un passage du domaine du discret d'un présent qui « ne cesse pas de se mouvoir, par bonds qui empiètent les uns sur les autres »- au domaine du continu constitué par un passé «pur » qui est « la coexistence toujours grandissante des niveaux du passé dans la synthèse passive $\mathrm{e}^{26} »$. Pour notre lecture de cette deuxième synthèse, nous nous concentrons ici sur cette idée de la limite : de la même façon que la méthode infinitésimale, basée sur la réduction progressive du changement vers une limite idéale de zéro, permet le passage du domaine du discret à celui du continu, chez Deleuze, cette même limite - dès lors que nous fixons sur le cas idéal où la durée du nouveau présent tend vers zéro - nous fournit une façon de saisir le mécanisme qui est au cœur de la deuxième synthèse du temps.

Pour décrire cette synthèse, Deleuze recourt à une série de trois paradoxes : la contemporanéité, la coexistence et la préexistence du passé ${ }^{27}$. Le premier - celui de la contemporanéité - est la solution deleuzienne au problème suivant: «si le passé attendait un nouveau présent pour se constituer comme passé, jamais l'ancien présent ne passerait ni le nouveau n'arriverait $^{28} »$. Bref, le temps resterait coincé dans un présent toujours gonflant. Pour surmonter ce problème, Deleuze avance que pour qu'un présent passe, il faut qu'il soit passé en même temps que présent. Dans quelles circonstances ce cas se vérifie-t-il ? C'est précisément à la limite idéale où un présent est sur le point de passer et où l'autre est sur le point de commencer, 
c'est-à-dire quand la durée du nouveau présent tend vers la limite de zéro. Nous pouvons trouver un exemple concret pour mieux comprendre ce premier paradoxe dans le concept de Anthropocène ${ }^{29}$ : bien que l'idée de cette nouvelle époque à l'Échelle des temps géologiques soit de plus en plus acceptée en raison de la croissance de l'espèce humaine et du bouleversement qu'elle provoque pour notre planète, il nous faut également rappeler que les scientifiques ne sont pas encore parvenus à un accord sur la marque stratigraphique qui formalise ce passage. Nous nous trouvons situés, par conséquent, dans une situation limitrophe entre deux époques - $1^{\prime}$ Holocène et l'Anthropocène - dont la première est à la fois déjà passée et toujours présente.

Mais qu'est-ce qui se passe avec le nouveau présent qui advient, l'autre moitié de ce passage? Si ce premier paradoxe nous explique pourquoi le présent passe, le deuxième- celui de la coexistence - porte sur la continuité $\mathrm{du}$ temps. Cette fois, Deleuze soutient que «tout le passé coexiste avec le nouveau présent par rapport auquel il est maintenant passé ${ }^{30} »$. Reprenant notre exemple de la naissance de l'Anthropocène, c'est le passé entier - tout le temps géologique : de l'Holocène à l'Hadéen et au-delà vers le Big-Bang qui coexiste avec ce présent qui advient. De la même manière que, dans la deuxième synthèse, le présent n'est qu'une dimension du passé, « la synthèse du tout temps entier ${ }^{31}$ ", l' Anthropocène n'est que la synthèse de tout le temps géologique qui le précède.

Ces deux paradoxes, qui représentent les deux perspectives de cette situation limitrophe où le temps passe, surviennent parce que le passé est doué de son être propre : il « est ». Le passé est présupposé à la fois par le présent qui passe (l'Holocène a besoin du temps géologique pour passer) et par le présent qui advient (l'Anthropocène a besoin du temps géologique pour contracter sa totalité et ainsi se constituer). De ces paradoxes Deleuze en déduit un troisième pour achever son explication : celui de la préexistence. La nature de ce passé pur et général, passé présupposé par le présent qui passe comme par celui qui advient, est a priori ; en d'autres termes, le passé préexiste au présent qui passe. C'est dans ce sens que la deuxième synthèse, celle du passé pur - cette accrétion toujours croissante dans laquelle tous les présents passent - est le fondement du temps.

Un des corollaires les plus importants de cette analyse des trois paradoxes de la deuxième synthèse est que, tout comme le passé et le futur formaient deux dimensions du présent dans la première synthèse, dans la deuxième, c'est le présent et le futur qui constituent des dimensions du passé. Deleuze explique ces deux dimensions en faisant référence à la métaphore bergsonienne du cône, où le présent n'est que le passé tout entier dans son état plus contracté qui coexiste avec soi «à une infinité de degrés de détente et de contraction divers, à une infinité de niveaux ${ }^{32}$ ». De la même façon, le futur se manifeste dans la liberté de «choisir» ces niveaux. Cependant, si, 
dans l'exposé de Deleuze, on peut bien arriver à comprendre ces deux dimensions pour le cas de l'être humain, sa pertinence en ce qui concerne les paysages et ses éléments constituants est moins intuitive. Sur ce point, l'analyse de Montebello sur l'univocité dans la philosophie de Bergson nous fournit une passerelle nous permettant de faire la connexion entre cet aspect bergsonien du passé chez Deleuze et d'autres types d'êtres. Montebello nous rappelle que ce qui distingue les trois strates de l'être, c'est précisément le niveau auquel chacun est capable de contracter le passé, c'est-à-dire le «pouvoir synthétique de faire passer le passé dans le présent ${ }^{33}$ ». On trouve ce pouvoir de manière très faible dans la matière. Le présent y possède « juste assez de durée, juste assez de passé pour une réaction immédiate ${ }^{34}$ », puisque dans la strate physique (dans l'eau d'un fleuve, par exemple), «la durée matérielle est précisément un rythme où le présent renaît sans cesse car, du passé, elle ne contracte rien de plus que ce qui est nécessaire à la réaction présente $^{35}$ ». En revanche, nous, les êtres humains, sommes situés à l'autre extrémité de cette échelle :

Ainsi émerge le nouveau visage de l'univers : durée diluée de la matière qui emporte continûment l'éternel cycle d'actions et de réactions; durée créatrice de la vie, immense virtualité qui éclate en formes au contact de la matière; durée synthétique de la conscience humaine qui se ressaisit librement au moment où tout son passé se coalise au présent dans la direction du futur; durée absolument libre enfin d'une supraconscience créatrice (durée hyper-contractée où tout le virtuel fait jaillir le réel) dont le cosmos surgit ${ }^{36}$.

Reprenant les exemples de la première synthèse, pensons à nouveau au désert du nord du Chili. Comme nous l'avons déjà vu, la présence de la vie dans cette région est très faible et, par conséquent, ce paysage manque de pouvoir synthétique qu'apportent les strates supérieures de l'être. Pendant des périodes très longues - de l'ordre de millions d'années - il n'a plus fait que réagir aux forces provoquées par les processus de subduction et d'autres sources d'activité. Le passage du temps de ce paysage n'est plus qu'un cycle permanent de réactions quasi instantanées sans aucune liberté. Cependant, si nous considérons la présence de la végétation, par exemple les oasis connectées par le réseau de sentiers dont nous avons parlé dans la partie précédente, la situation commence à changer. Il y a une augmentation du pouvoir synthétique - bien qu'il reste faible - où le rythme de la croissance et de la reproduction des plantes recouvre une durée plus ample, comprenant les cycles des saisons et de la reproduction des plantes, fournissant ainsi au paysage le degré de liberté nécessaire pour s'adapter aux changements du climat en variant la composition ou position des espèces. Comme le souligne Montebello, c'est précisément cette augmentation de pouvoir synthétique qui distingue les deux strates supérieures de l'être (physiologique et psychologique) de la strate physique. Alors, si nous rajoutons des habitants 
humains au paysage, ce pouvoir synthétique augmente de nouveau de façon très importante grâce à la liberté qu'y apporte la conscience humaine. Cet élément psychologique permet aux paysages d'évoluer de nouvelles et diverses façons. Cela peut prendre la forme d'une évolution plus douce, par exemple dans les modes d'habitation des peuples autochtones ${ }^{37}$, comme dans le cas des géoglyphes et du réseau de sentiers que nous avons traités dans la première synthèse, ou bien d'une évolution plus brutale, comme l'exploitation minière à grande échelle qui commence avec l'arrivée des colons européens ${ }^{38}$. Quoi qu'il en soit, le pouvoir synthétique humain de saisir un présent plus ample a permis le façonnement du paysage à travers la création de ces éléments.

Il y a la tentation, particulièrement dans ce dernier cas, de ne se concentrer que sur l'élément humain, mais chaque paysage est défini par une chronopédie unique, composée d'êtres situés sur les trois strates, chaque être ayant sa temporalité et son rythme propres. Notre conscience de cette chronopédie nous situe à l'extrémité de l'échelle présentée par Montebello et notre capacité de vivre un présent ample comme une dimension du passé deleuzien nous fournit un degré de liberté plus élevé. Cependant, il s'agit de comprendre notre place dans la totalité de cette composition, la manière dont les êtres humains font partie de ces chronopédies. Nous avons déjà commencé à voir cet aspect dans l'exposition de la deuxième synthèse et nous allons l'approfondir dans notre analyse de la troisième.

\section{L'effondrement : l'avenir}

Dans les deux parties précédentes, nous avons vu deux synthèses qui constituent respectivement la fondation et le fondement du temps deleuzien. Cependant, toutes les deux - la contraction des habitudes au travers de la contemplation des événements dans le présent et le passage de ce présent au passé - étaient passives. Il manque toujours une explication de la capacité de produire le changement, créant le nouveau et façonnant l'avenir. C'est la tâche de la troisième synthèse deleuzienne. Dans un certain sens, cette synthèse active est la plus complexe des trois, puisque sa présentation dans les termes du sujet humain, avec recours aux dramatis personæ d'CEdipe et de Hamlet, la rend la moins intuitive en ce qui concerne son application aux autres strates de l'être. Cependant, nous verrons ici comment une lecture qui libère cette synthèse du sujet humain nous permet de voir comment le temps deleuzien pourrait apporter à une métaphysique qui cherche à résoudre l'écart entre l'être humain et la nature.

Au niveau le plus profond, on peut penser la troisième synthèse du temps comme le point culminant d'une évolution fondamentale dans la pensée occidentale depuis plusieurs siècles. L'analyse de Deleuze remonte à Descartes et au cogito cartésien, qui divise le sujet humain entre la détermination (je pense) et de l'indéterminé (je suis). Cependant, ce cogito, 
remarque Deleuze, ne laisse aucune place au temps : «Descartes ne concluait $q u^{\prime}$ à force de réduire le Cogito à l'instant, et d'expulser le temps, de le confier à Dieu dans l'opération de la création continuée ${ }^{39}$. » Cette temporalité divine durera plus de deux siècles, jusqu'à Kant et sa découverte du transcendantal, moment qui, selon Deleuze, ne représente rien de moins qu'une vraie « révolution copernicienne » dans notre pensée. Chez Kant, la temporalité cartésienne fondée sur la garantie divine de la cohérence du sujet cède la place, dans la «mort spéculative de $\mathrm{Dieu}^{40}$ ", à une différence interne. Au binôme cartésien de la détermination et l'indéterminé, Kant ajoute un nouveau terme, le déterminable, qui définit la forme de cette nouvelle temporalité. Dans son analyse, Deleuze souligne comment, ce faisant, Kant ouvre une "fêlure » entre l'activité du sujet et sa passivité : " la spontanéité dont j'ai conscience dans le Je pense ne peut pas être comprise comme l'attribut d'un être substantiel et spontané, mais seulement comme l'affection d'un moi passif qui sent que sa propre pensée, sa propre intelligence, ce par quoi il dit JE, s'exerce en lui et sur lui, non pas par lui ${ }^{41}$. » Or, conclut-il, bien que Kant ouvre cette fêlure dans le sujet et qu'il découvre la forme du temps, il ne poursuit pas cette initiative jusqu'à ses dernières conséquences, là où le moi reste «seulement défini par la réceptivité, ne possédant à ce titre aucun pouvoir de synthèse ${ }^{42}$ ». Dans sa troisième synthèse, Deleuze se charge de repenser cette fêlure et de comprendre l'imbrication de l'activité du sujet et de la passivité du moi qui se trouvent au cœur du temps.

Mais cela pose la question de la manière d'interpréter ce sujet actif et ce moi passif au-delà du monde humain. Comme Williams le souligne dans son analyse du temps deleuzien, pour bien comprendre cette troisième synthèse, il ne faut pas perdre de vue que le mot «actif ne veut pas forcément dire déployé par un sujet actif mais désigne plutôt un processus qui va d'un ensemble plus grand d'éléments à un ensemble représentatif plus petit », de sorte que « l'activité veut dire la sélection par l'introduction d'une différence nouvelle, la passivité veut dire déterminé hors du contrôle de l'activité, le sujet désigne un processus de sélection, et fêler veut exprimer la dissolution du sujet par les synthèses pour lesquelles il est passif ${ }^{43}$ ». Considérons, par exemple, le cas des tremblements de terre, comme ceux qui sont si fréquents dans les paysages que nous avons évoqués ci-dessus. La détermination prend la forme du déplacement ou de la reconfiguration de la terre et l'indétermination concerne l'être même du paysage : le paysage se déplace, donc il est. Lors de ces tremblements de terre, le relief du paysage est reconfiguré et une nouvelle configuration du terrain est choisie parmi toutes les possibles reconfigurations. Cependant, cette activité est à la fois le produit passif des forces sismiques, des tensions et frictions des plaques tectoniques, de la pression exercée par et sur la croûte, et - dans un contexte moderne - le produit passif des activités provenant de l'habitation humaine, telles que la fracturation hydraulique pratiquée pour libérer des hydrocarbures ou la construction de barrages qui changent subtilement les pressions sur la croûte terrestre. De la même façon, dans le cas de la forêt, nous pouvons dire que le 
paysage pousse, donc qu'il est, où cette activité, cette poussée, est le produit des synthèses passives comme celles définies par l'orographie et l'hydrographie de la région. Finalement, dans le cas d'un paysage avec activité humaine, par exemple l'extraction des minéraux cuprifères de couleur turquoise par les peuples préhispaniques qui traversaient les chemins du désert, le paysage se fragmente ou se désintègre, donc il est ; cette fragmentation et cette désintégration sont le produit des innombrables synthèses passives qui les déterminent $t^{44}$.

La fêlure associée à cette imbrication de l'activité du sujet et de la passivité du moi marque aussi une rupture de la continuité du temps à cause du changement introduit par l'action du sujet. Deleuze explique cette rupture de plusieurs manières : c'est le temps « hors de ces gonds » de Hamlet ; $c^{\prime}$ est le temps de la «césure» de Hölderlin qui ne «rime»plus; c'est le déroulement de la continuité du cercle «trop simple» de la ressemblance et de la représentation du Même ${ }^{45}$. Quoi qu'il en soit, cette rupture définit un ordre qui est déterminé par la discordance entre l'avant et l'après. Cet ordre définit la forme pure et vide du temps qui n'est rien de moins que la forme du changement lui-même. Voici la troisième synthèse : une synthèse statique et $a$ priori qui distribue l'avant et l'après autour de la discordance de la fêlure ; statique parce que « la forme du changement ne change pas » et a priori parce que, comme dans la deuxième synthèse, il s'agit aussi du cas liminal et insaisissable du devenir ${ }^{46}$.

Cette discordance définit à la fois un ensemble et une série : un ensemble parce qu'elle rassemble la totalité du temps de sorte qu'elle est « adéquate au temps tout entier »; une série parce que dans le premier membre de cet ensemble, le temps est, dans un certain sens, «trop grand ${ }^{47}$ ». Pour mieux comprendre ces deux termes, suivons la lecture mathématique de Voss de la troisième synthèse en termes des «coupures" de Dedekind ${ }^{48}$. À titre d'exemple, le nombre irrationnel $\sqrt{2}$ coupe l'ensemble des nombre réels en deux sous-ensembles: dans le premier, $A$, les membres sont définis par l'inégalité $x^{2}<2$; dans le deuxième, $B$, ils sont définis par l'inégalité $x^{2}>2$. Cela nous fournit un ensemble avec trois membres (A, $\sqrt{2}, B)$, un ensemble qui comprend la totalité des nombres réels et qui est à la fois une série déterminée autour de l'inégalité introduite par son deuxième membre. C'est dans ce sens que nous pouvons comprendre l'ensemble et la série de la troisième synthèse: elle rassemble le temps en sa totalité avec ces trois éléments et les met dans une série qui respecte l'ordre a priori du temps. Ainsi, de la même façon que la première synthèse contient le passé et le futur comme dimensions et que le deuxième contient le présent et le futur, dans la troisième, le passé et le présent sont des dimensions du nouveau futur. Il y a un passé où l'action du présent est toujours « trop grande » pour le moi (dans le même sens que $\sqrt{2}$ est trop grand pour le premier sous-ensemble dans notre exemple mathématique) et il y a un présent qui prend la forme d'une 
métamorphose définie par le «devenir-égal à l'action ${ }^{49}$ ». Reprenant notre exemple du tremblement du terre, il y a un temps où l'image de la nouvelle configuration du terrain est encore «trop grande » pour le paysage. Celui-ci n'est pas prêt : les conditions ne sont pas encore là pour que le changement ait lieu. Il faut attendre un peu plus pour que l'accumulation des pressions tectoniques atteigne leur seuil critique. Il y a aussi le présent : le moment du tremblement de terre lui-même où, franchi ce seuil, le paysage subit une métamorphose où il devient égal à l'image de la nouvelle configuration de la terre. Mais ce sujet qui émerge pour réaliser l'action, pour effectuer le changement au monde, est à la fois dissous par les synthèses passives qui le définissent (ici, par exemple, les courants du magma sur lesquels flottent les plaques tectoniques), de façon à ce que ni la condition du passé ni l'agent de la métamorphose ne puissent revenir ${ }^{50}$. Le paysage est désormais irrévocablement autre. Tel est le bouleversement du fondement de la troisième synthèse, le sans-fond ou $1^{\prime}$ « universel effondrement » propre de l'avenir ${ }^{51}$.

Si la deuxième synthèse du temps deleuzien était bergsonienne en raison de son traitement du passé, le traitement de l'activité et de l'avenir dans la troisième est marqué par un profond courant nietzschéen. Dans une note additionnelle à son exposition de la troisième synthèse, Deleuze attire notre attention sur la manière dont la temporalité de Ainsi parlait Zarathoustra imite celle de la troisième synthèse. Zarathoustra vit la plupart du temps dans le passé, où l'idée de l'éternel retour est «trop grande » pour lui, dans l'attente de la métamorphose où il devient «capable » de la révélation de l'éternel retour $^{52}$. Même si cette révélation n'a jamais été écrite par Nietzsche, c'est dans l'idée de l'avenir comme éternel retour que nous trouvons le point capital de la troisième synthèse :

L'éternel retour n'affecte que le nouveau, c'est-à-dire ce qui est produit sous la condition du défaut et par l'intermédiaire de la métamorphose. Mais il ne fait revenir ni la condition ni l'agent ; au contraire, il les expulse, il les renie de toute sa force centrifuge. [...] Il est la répétition par excès, qui ne laisse rien subsister du défaut ni du devenir-égal. Il est lui-même le nouveau, toute la nouveauté. Il est lui seul le troisième temps de la série, l'avenir en tant que tel. $^{53}$

C'est précisément dans l'éternel retour nietzschéen que Deleuze trouve la réponse à la fêlure ouverte par la philosophie kantienne. Cependant, au niveau plus profond, il y a peut-être un autre point de convergence moins évident avec la pensée nietzschéenne. Au début de cette explication de la troisième synthèse, nous nous sommes servis des définitions fournies par Williams de l'activité comme «sélection par l'introduction de différences nouvelles » et du sujet comme « un processus de sélection » pour libérer la troisième synthèse du sujet humain ${ }^{54}$. Cependant, on peut également constater que, chez Nietzsche, la volonté de puissance - la pierre angulaire de sa philosophie, avec son schéma tripartite du sentir-penser-vouloir - ouvre 
une voie pour penser la troisième synthèse en termes d'êtres non humains et d'activité. Comme Montebello le démontre, pour Nietzsche, même dans la strate physique, "la pensée" et "la sensation" sont toujours "les signes de quelque processus" »; ils sont "les signes de processus relationnels de domination et de conquête, d'opposition et de résistance, d'explosion et de changement de direction ${ }^{55}$ ». Comme la deuxième synthèse, 1'univocité de la pensée bergsonienne nous avait permis d'étendre le temps deleuzien aux autres strates de l'être, pour la troisième, c'est l'univocité chez Nietzsche qui nous le permet.

Pour conclure cette explication de la troisième synthèse, reprenons l'exemple des géoglyphes dans le désert. Même si c'est nous, les êtres humains, qui sommes intervenus sur le paysage, la troisième synthèse nous apprend que c'est une perspective incomplète. En revanche, il y a une perspective non anthropocentrique qui nous permet de mieux comprendre ce changement et qui nous offre une perspective beaucoup plus riche du monde : selon ce point de vue, c'est le paysage lui-même qui a changé, qui a évolué et qui s'est exprimé à travers des géoglyphes. Si les habitants préhispaniques de ce paysage ont su créer les géoglyphes, c'est parce qu'ils étaient liés d'une façon très intime et irrévocable à ce paysage: les traces des chemins sont déterminées par l'hydrographie du fleuve Loa ; les formes zoomorphiques de certains des dessins reflètent la faune soutenue par le paysage ; la pratique même de créer ces signes sur les collines a été définie par des croyances déjà perdues à l'époque mais qui refléteront certainement le milieu habité par ces peuples autochtones. Ce sont deux perspectives sur le monde : l'une, propre au monde moderne, qui situe les humains en dehors d'un paysage inerte et qui est le produit d'une nature mécanisée et maîtrisée par les humains ; l'autre provient plutôt d'une chronopédie, d'une composition des rythmes vivants des divers êtres qui comprennent le paysage où les humains ne sont qu'une partie infime et transitoire de ce vaste ensemble.

\section{Conclusion}

Nous avons vu comment le temps deleuzien est fondé sur trois modes de répétition, chacun abordé par sa synthèse respective. La première synthèse (le présent vivant) est la fondation du temps qui contracte les instants répétés de présents discrets, fournissant les habitudes qui constituent l'être. Nous avons vu comment il est possible de penser les montagnes, les forêts et même les traces laissées par les humains comme des habitudes de l'être d'un paysage. La deuxième synthèse (le passé pur) est le fondement du temps, qui garantit sa continuité et son passage. Grâce au courant bergsonien qui sous-tend cette synthèse, nous avons vu comment ce devenir-passé d'un présent est étroitement lié au pouvoir synthétique des différentes strates de l'être et à sa « conscience » de la durée du présent, de la durée diluée et du pouvoir faible des êtres inorganiques jusqu'au pouvoir synthétique formidable des êtres 
humains. Finalement, la troisième synthèse concerne la répétition ou l'éternel retour de la nouveauté, rendant possible le changement et l'avenir. Dans cette dernière synthèse, Deleuze trace une évolution de la temporalité de la pensée occidentale du cogito cartésien, où Dieu a garanti la cohérence du sujet dans le temps, à travers la révolution copernicienne de Kant qui a fêlé le sujet et introduit le temps en son sein, et jusqu'aux idées de Nietzsche que Deleuze emploie pour réaliser les dernières conséquences de cette fêlure: une philosophie du temps basée sur la répétition de la différence elle-même. Dans cette dernière synthèse, nous avons appris comment penser les changements d'un paysage comme le produit de l'interaction entre le sujet actif et les synthèses passives qui définissent son activité. Cette interaction est au cœur de la (re)découverte du dynamisme du monde naturel et se débarrasse du mythe d'une nature mécanisée, inerte et ouverte à la conquête humaine, mythe qui a marqué de façon si profonde notre modernité.

En explorant la façon dont le temps deleuzien peut servir à penser les paysages, cet article a cherché à démontrer comment, pris ensemble, ces trois modes de répétition - le « répétiteur » du présent, la « répétition même » du passé et le « répété » de l'avenir - constituent une métaphysique capable de soutenir une pensée où l'être humain n'est plus au centre de la nature, une pensée anthropomorphique dans son univocité mais sans être anthropocentrique. Dans ce sens, le temps deleuzien constitue un événement avec son propre avant et son propre après, avec sa propre métamorphose où le point culminant de l'effacement de l'homme annoncé par Foucault ${ }^{56}$ quelques années plus tôt marque un devenir égal à l'avenir de l'Anthropocène. Dans chacune des synthèses, en cherchant à penser les paysages en termes temporels, nous avons vu comment la composition des temps des différents êtres qui font partie d'un paysage - chacun avec son propre rythme - constitue une chronopédie. Le manque de conscience de notre position dans ces chronopédies et notre désynchronisation avec les paysages que nous habitons en conséquence ont constitué les conditions de cette métamorphose. Le défi consistant à apprendre à les lire et à nous remettre en synchronisation avec nos habitats définira le sort de notre espèce dans ce nouvel avenir.

${ }^{1}$ Les débats sur le rôle qu'occupent les humains dans le monde naturel ne sont pas un phénomène nouveau : Ernest Garcia montre qu'au moins pendant le XVIII siècle, les idées sur les limites de la croissance humaine avaient été discutées entre des 
intellectuels, comme Nicolas de Condorcet et Thomas Robert Malthus. Voir « Le XVIIIe siècle et les limites de la croissance ", L'Ecologiste 17, no 3 (2016) : 37-41.

2 Ce dualisme est traité par Alexandre Federau dans Pour une philosophie de l'Anthropocène (Paris: Presses Universitaires de France, 2017), 17-18. Marco Altamirano offre une analyse approfondie du rôle de la pensée cartésienne dans la création de cette «bifurcation" de la nature dans Time, Technology and Environment: An Essay on the Philosophy of Nature (Édimbourg : Edinburgh University Press, 2019).

${ }^{3}$ Pierre Montebello, L'autre métaphysique (Dijon : Les Presses du réel, 2015), 3-6.

${ }^{4}$ Pierre Montebello, Métaphysiques cosmomorphes. La fin du monde humain (Dijon : Les Presses du réel, 2015), 5.

${ }^{5}$ Gilles Deleuze, Différence et répétition (Paris : Presses Universitaires de France, 1968), 96-168.

${ }^{6}$ Pierre Montebello, L'autre métaphysique, 6.

${ }^{7}$ Néologisme inventé par Michel Serres dans son livre L'Incandescent, où il pose la question suivante : «Dans quelle étendue se développe-t-il [le Grand Récit de notre Univers] ? Dans un paysage. Les sciences décrivent aujourd'hui et tentent de rapiécer des morceaux de ces paysages, un peu comme les sciences humaines désespèrent de recoudre les cultures entre elles »; L'Incandescent (Paris : Le Pommier, 2003), 4647. Serres propose le concept de " chronopédie " pour rassembler ces morceaux, chacun avec sa durée et son rythme différent : "Dans ce paysage nous lisons une chronopédie »; L'Incandescent (Paris : Le Pommier, 2003), 46-47.

${ }^{8}$ Deleuze, Différence et répétition, 96-168. Même si Deleuze revient sur le sujet du temps dans Logique du sens (Paris : Éditions de Minuit, 1969) ; et Cinéma 2 : L'imagetemps (Paris : Éditions de Minuit, 1985) ; ici nous nous limiterons aux trois synthèses de Différence et répétition qui constituent le cœur de sa métaphysique. Il touche également à la géologie et aux paysages avec Félix Guattari dans Mille plateaux (Paris : Éditions de Minuit, 1980), notamment dans les chapitres « Année zéro Visagité » et «10 000 av. J .-C. - La géologie de la morale ». Au ce sujet, voir aussi Mark Bonata et John Protevi, Deleuze and Geophilosophy: A Guide and Glossary (Édimbourg : Edinburgh University Press, 2006).

9 Exemples basés sur les pratiques d' «écriture créative» de l'auteur. Voir, par exemple, James Kelly, "From sea to source: The journey of the Biobío River ", à paraître dans Antennae: The journal of nature in visual culture.

10 James Williams caractérise la pensée de Deleuze sur le temps comme « a philosophy of time at the crossroads of the history of philosophy, of modern sciences and of 
contemporary problems about time "; Gilles Deleuze's Philosophy of Time: A Critical Introduction and Guide (Édimbourg : Edinburgh University Press, 2011), 1-2.

${ }^{11}$ Williams, Gilles Deleuze's Philosophy of Time, 51.

12 Deleuze, Différence et répétition, 101.

13 Deleuze, Différence et répétition, 99, 101-2.

${ }^{14}$ Deleuze, Différence et répétition, 99, 101-2.

${ }^{15}$ Deleuze, Différence et répétition, 99, 101-2.

${ }^{16}$ Ces trois strates de l'être figurent dans l'analyse de Montebello de l'univocité des philosophies de Nietzsche et Bergson dans L'autre métaphysique. Il serait intéressant d'analyser plus dans le détail la correspondance entre les trois strates de l'analyse de Montebello et la série géosphère-biosphère-noosphère. Pour une discussion sur la biosphère et la noosphère, voir Federau, Pour une philosophie de l'Anthropocène, $168-80$; et Vladimir Vernadsky dans "The biosphere and the noosphere, " American Scientist 33, n० 1 (1945) : 1-12.

17 Vernadsky, «The biosphere and the noosphere, »1-12.

18 Pour plus d'informations sur les géoglyphes au nord du Chili, voir Gonzalo Pimentel, Mariana Ugarte, Francisco Gallardo, José F. Blanco et Claudia Montero, « Chug-Chug en el contexto de la movilidad internodal prehispánica en el desierto de atacama, Chile ", Chungara: Revista de Antropología Chilena 49, nº 4 (2017) : 483-510.

${ }^{19}$ Deleuze, Différence et répétition, 105.

${ }^{20}$ Deleuze, Différence et répétition, 102-3.

21 Deleuze, Différence et répétition, 105.

22 Deleuze, Différence et répétition, 108.

${ }^{23}$ Gilles Deleuze, Le bergsonisme (Paris : Presses Universitaires de France, 1966), 50 52.

24 Deleuze, Le bergsonisme, 50-52.

${ }^{25} \mathrm{Au}$ fond, c'est un paradoxe du mouvement qui hante la pensée de Bergson ainsi que celle de Deleuze. Michel Souriau, l'œuvre que Deleuze cite dans sa présentation des trois synthèses du temps, décrit le calcul infinitésimal comme « le procédé magique du contact entre l'esprit et les chose, " rajoutant que " à sa base se trouve le temps continu»; Le temps (Paris: Librairie Félix Alcan, 1937), 40. Jean Milet écrit également de manière approfondie sur le calcul différentiel dans la philosophie de Bergson; Bergson et le calcul infinitésimal ou la raison et le temps (Paris: Presses Universitaires de France, 1974) ; et dans Ontologie de la différence : une exploration du champ épistémologique (Paris: Beauchesne, 2006).

${ }^{26}$ Gilles Deleuze, Le bergsonisme, 113. 
27 Williams aussi analyse ces paradoxes dans Gilles Deleuze's Philosophy of Time, 6467 ; ainsi que Alia Al-Saji dans « The Memory of Another Past: Bergson, Deleuze and A New Theory Of Time, » Continental Philosophy Review 37 (2004) : 203-39.

${ }^{28}$ Deleuze, Différence et répétition, 111.

${ }^{29}$ Pour un résumé des débats sur l’Anthropocène, voir Federau, Pour une philosophie de l'Anthropocène, $121 \mathrm{ff}$.

30 Deleuze, Différence et répétition, 111.

${ }^{31}$ Deleuze, Différence et répétition, 111.

32 Deleuze, Différence et répétition, 112.

33 Montebello, L'autre métaphysique, 239. Dans sa présentation, Deleuze nous rappelle que la durée du présent est « variable suivant les espèces, les individus, les organismes et les parties d'organisme considérées »; Différence et répétition, 105.

${ }^{34}$ Montebello, L'autre métaphysique, 236.

${ }^{35}$ Montebello, L'autre métaphysique, 236.

${ }^{36}$ Montebello, L'autre métaphysique, 240-41.

37 Levis et al., par exemple, analysent en profondeur les effets des peuples autochtones sur l'évolution des paysages précolombiens dans le contexte sudaméricain, démontrant comment leur présence a produit des changements prolongés sur les forêts de l'Amazone: «Persistent effects of pre-Columbian plant domestication on Amazonian forest composition. » ; Science 355, nº 6328 (2017) : 92531.

38 Dans le cas de l'exploitation minière à grande échelle, nous voyons que cette intervention n'est pas seulement soutenue par la technologie mais aussi par l'évolution de notre conscience temporelle avec la découverte du temps profond de la géologie et notre capacité de comprendre les processus qui ont laissé les dépôts de métaux et de minéraux.

${ }^{39}$ Deleuze, Différence et répétition, 116-17.

40 Deleuze, Différence et répétition, 116-17.

${ }^{41}$ Deleuze, Différence et répétition, 116-17.

42 Deleuze, Différence et répétition, 116-17.

${ }^{43}$ Williams, Gilles Deleuze's Philosophy of Time, 57, 90.

44 Du point du vue du langage, on peut constater que les verbes employés dans ces exemples (déplacer, pousser, fragmenter) ne donnent qu'une faible approximation de la richesse de cette activité. Cela pose la question de la manière de traduire dans nos langues humaines cette « écriture » de la Terre. Aurions-nous besoin, comme l'a suggéré Michel Serres d’une nouvelle langue ?; Biogée (Brest : Éditions dialogues, 
2010), 170. Ce thème est traité en profondeur par Eduardo Kohn dans Comment pensent les forêts : Vers une anthropologie au-delà de l'humain (Bruxelles : Zones sensibles, 2017).

45 Deleuze, Différence et répétition, 119-20.

${ }^{46}$ Deleuze approfondit ce cas liminal du devenir dans Logique du sens.

${ }^{47}$ Deleuze, Différence et répétition, 120.

${ }^{48}$ Daniela Voss, «Deleuze's Third Synthesis of Time, » Deleuze Studies 7, n 2 (2013) : 194-216. Deleuze parle de la coupure de Dedekind dans le quatrième chapitre de Différence et répétition, 223.

${ }^{49}$ Deleuze, Différence et répétition, 121.

${ }^{50}$ Deleuze, Différence et répétition, 122.

51 Deleuze, Différence et répétition, 123.

52 Deleuze, Différence et répétition, 124.

53 Deleuze, Différence et répétition, 122.

54 Williams, Gilles Deleuze's Philosophy of Time, 57, 90.

${ }^{55}$ Montebello, Pierre, Nietzsche. Fidélité à la Terre (Paris : CNRS Éditions, 2019), 243-48.

${ }^{56}$ Michel Foucault, Les mots et les choses : une archéologie des sciences humaines (Paris : Gallimard, 1966), 398. 